\title{
Confiabilidad aplicada al diseño geotécnico de un muro de contención
}

\section{Reliability applied to the geotechnical design of a retaining wall}

\section{Felipe Prada ${ }^{1}$, Alfonso Ramos $^{2}$, Diana Solaque ${ }^{3}$ y Bernardo Caicedo ${ }^{4}$}

${ }^{1}$ Instituto de Mecánica de Suelos y Rocas. Karlsruhe Institute of Technology, Engler-Bunte-Ring 14, 76131 Karlsruhe, Alemania, felipe.prada@kit.edu

${ }^{2}$ Instituto Geofísico, Facultad de Ingeniería. Pontificia Universidad Javeriana. Carrera 7 No. 42 - 27 Piso 7. Bogotá, Colombia, a-ramos@javeriana.edu.co

${ }^{3}$ INGETEC S.A. Carrera 6 No.30A - 30. Bogotá, Colombia, dianasolaque@ingetec.com.co

${ }^{4}$ Facultad de Ingeniería. Universidad de Los Andes. Edificio Mario Laserna Cra 1 E No 19A - 40, Oficina ML-775. Bogotá, Colombia, bcaicedo@uniandes.edu.co

La práctica actual de diseño en la ingeniería civil concentra sus esfuerzos en cuantificar el factor de seguridad para definir si una estructura es segura o no. Sin embargo el factor de seguridad por sí solo no da cuenta de la probabilidad de falla que tiene una estructura. Esta práctica está aún más arraigada en el campo de la geotecnia. Este artículo muestra tres métodos para estimar las probabilidades de falla de estructuras geotécnicas, el método de primer orden segundo momento, estimación puntual y simulaciones de Monte Carlo. Los tres métodos se aplican a la estimación de la probabilidad de falla de un muro de gravedad adyacente a un talud con lo que se tiene la posibilidad de determinar el ancho óptimo del muro de contención en función de los costos asociados. Adicionalmente fue necesario derivar los factores $N_{c} y N_{q}$ para capacidad portante que tuvieran en cuenta el efecto de la superficie inclinada del terreno, con el fin que pudieran ser usados en los métodos de análisis en confiabilidad. Finalmente se comparan los resultados obtenidos por los tres métodos y se discute la conveniencia del análisis de confiabilidad para el caso estudiado.

Palabras clave: confiabilidad, estructuras geotécnicas, probabilidad de falla

\section{Introducción}

La geotecnia puede considerarse como una de las áreas de la Ingeniería Civil más complejas y en donde más simplificaciones se hacen para entender y poder reproducir el comportamiento del suelo. Diferentes metodologías han surgido con el fin de mejorar las aproximaciones empíricas basadas principalmente en el método observacional que permitieron el desarrollo inicial de la geotecnia. Dentro de estas metodologías se encuentra el diseño basado en la confiabilidad en el cual las propiedades del suelo y las solicitaciones se caracterizan como funciones probabilísticas. La variabilidad inherente de las propiedades geomecánicas de los suelos y de las
The daily practice of designing civil engineering projects focuses its efforts on quantifying the factor of safety to ascertain whether a structure is safe or not. However, the factor of safety in itself does not reflect the probability of failure of the designed structure. This practice is deeply rooted within the geotechnical engineering field. This article shows three methods; first order second moment, point estimation, and Monte Carlo simulations. These three methods are used to estimate the probability of failure of a geotechnical structure, namely a gravity retaining wall adjacent to a slope. The probabilistic analysis allows to determine the optimal base width of the wall as a function of the construction costs. A geometrical deduction of the bearing capacity factors $N_{c}$ and $N_{q}$ for the slope condition is presented. Finally, results obtained from the three analysed methods are compared and some recommendations for the convenience of reliability analysis are discussed.

Keywords: reliability, geotechnical structures, probability of failure

cargas aplicadas hace que los diseños desarrollados en términos determinísticos no sean óptimos en términos económicos y a la vez no se tenga una idea de la cuantificación de la incertidumbre asociada. Diseños basados en confiabilidad permiten analizar de mejor forma el desempeño de una estructura geotécnica en términos de probabilidades de falla en vez de los tradicionales factores de seguridad.

En la Ingeniería Geotécnica se debe propender porque los diseños cumplan requisitos de funcionalidad, seguridad, resistencia y economía dentro de unos límites razonables. Para lograr ese propósito lo usual es transformar las condiciones de trabajo de las cimentaciones 
en modelos matemáticos, algunas veces supremamente simplificados, donde se idealizan tanto los parámetros geomecánicos del terreno de fundación como las acciones impuestas a las cimentaciones. Por consiguiente, a pesar de que el diseño de una cimentación sea resultado de los mejores estimativos que puedan hacerse sobre el comportamiento del terreno de fundación y la superestructura, nunca habrá garantía absoluta de que una vez construida se comporte estrictamente dentro de los niveles de estabilidad y deformación considerados en la modelación analítica.

La causa de este problema suele estar relacionada con la combinación de los siguientes factores (Berdugo, 2000):

-La incertidumbre sobre la representatividad de la caracterización geotécnica

-La incertidumbre sobre la naturaleza y magnitud real de las acciones impuestas a la cimentación y su variación espacial en el tiempo

-La incertidumbre sobre la validez de los métodos de análisis

-La incertidumbre sobre los efectos de los procesos de construcción sobre el comportamiento de la cimentación

-La incertidumbre sobre la tolerancia de la cimentación a las acciones impuestas por la superestructura y el terreno de fundación

Para que un diseño tenga en cuenta los requisitos anteriormente mencionados de forma simultánea y poder atacar las múltiples incertidumbres inherentes al proceso, se recomienda implementar el análisis de confiabilidad en cimentaciones. Del correcto uso que se le dé a las formulaciones probabilísticas $\mathrm{y}$ de riesgo depende que las decisiones que se tomen sean correctamente basadas en criterios objetivos ( $v g$. costo, seguridad).

\section{Función de estado límite o función de desempeño}

Para poder hacer estimativos de confiabilidad es necesario tener una función de estado límite o función de desempeño. Una función de estado límite es aquella que delimita un dominio seguro de uno no seguro. En geotecnia, usualmente se usa como funciones de estado límite el factor de seguridad (FS) y el margen de seguridad (MS) definidos como:

$$
F S=\frac{\text { Resistencia }}{\text { Carga }}
$$

Si se aplica a cimentaciones, tiene que:

$$
\begin{gathered}
F S=\frac{\text { Capacidad Portante }}{\text { Carga }} \\
M S=\text { Capacidad Portante - Carga }
\end{gathered}
$$

Tanto FS como MS dependen, entre otras, de las propiedades mecánicas del suelo, que a su vez serán tratadas como variables probabilísticas. Es decir, que la realización de la variable está caracterizada por una función de densidad de probabilidad con parámetros de dicha función, por ejemplo la media y la desviación estándar para una variable que se distribuye normal o lognormal.

En este artículo se utilizará el factor de seguridad FS como función de estado límite $g\left(x_{i}\right)$. $g$ es la función de estado límite y $x_{i}$ son las variables de las que depende la función de estado límite (cohesión, fricción, peso unitario, etc).

\section{Métodos de análisis de confiabilidad}

Existen diferentes técnicas para aproximarse al índice de confiabilidad y la probabilidad de falla $\left(p_{f}\right)$ de un problema geotécnico. Los métodos usados en este trabajo son: método de primer orden segundo momento, método de estimación puntual y simulaciones de Monte Carlo.

\section{Método de Primer Orden Segundo Momento - series de Taylor}

Este método usa los primeros términos de una expansión de la serie de Taylor de la función de desempeño para estimar el valor esperado y la varianza de la función de desempeño. Este método supone que $\left(x_{i}-\mu x_{i}\right)$ de cada una de las variables aleatorias es pequeña $\left(x_{i}=\right.$ variable, $\mu x_{i}=$ valor esperado de la variable) por lo que el cuadrado o potencias mayores son aún más pequeñas y se pueden ignorar. Es llamado de segundo momento porque la varianza es de la forma de segundo momento y es el resultado estadístico de mayor orden usado en el análisis. El valor esperado de la función de desempeño $E[g]$ se obtiene como:

$$
E[g] \approx g\left(\mu x_{1}, \mu x_{2}, \ldots \mu x_{n}\right)
$$

es decir, el valor esperado de la función de desempeño $E[g]$ se obtiene evaluando el valor medio de cada variable 
aleatoria $\mu x_{i}$ en la función de desempeño $g$.

Suponiendo que las variables aleatorias son independientes entre si, la varianza de la función de desempeño $V[g]$ queda como:

$$
V[g]=\sum_{i=1}^{n}\left[\frac{\partial g\left(x_{1}, \ldots x_{n}\right)}{\partial x_{i}} \|_{\mu x_{1}, \mu x_{2}, \ldots \mu x_{n}}\right]^{2} V\left[x_{i}\right]
$$

Si el número de variables aleatorias es $n$, este método requiere evaluar $n$ derivadas parciales de la función de desempeño o desarrollar una aproximación numérica usando la evaluación en $2 n+1$ puntos (Baecher y Christian, 2003).

\section{Método de estimación puntual}

Rosenblueth (1975) propuso un método simple para obtener los momentos de la función de desempeño por medio de la evaluación de la función de desempeño en un juego de puntos discretos escogidos. La aproximación de Rosenblueth es una aplicación del método de la cuadratura de Gauss para ubicar las coordenadas de los puntos óptimos para evaluar una integral y los pesos correspondientes a los puntos. En este caso, los puntos óptimos para evaluar los momentos de la función de densidad de probabilidad para cada variable aleatoria es el valor promedio \pm la desviación estándar. Si el problema tiene $n$ variables aleatorias, se tendrán $2^{n}$ combinaciones de la función de desempeño. Con las $2^{n}$ funciones de desempeño se puede calcular un valor esperado de la función de desempeño $E[g]$ y la varianza de la misma $V[g]$. Una de las desventajas del método es que al tener que evaluar $2^{n}$ veces la función de desempeño, puede llegar a ser dispendioso cuando el número de variables aleatorias es grande (Baecher y Christian, 2003).

\section{Simulación de Monte Carlo}

Este método utiliza el muestreo aleatorio para simular artificialmente el comportamiento de un sistema (Sánchez, 2005). En esta aproximación el analista crea un gran número de juegos de valores generados aleatoriamente para los parámetros probabilísticos y se calcula la función de desempeño para cada juego de datos de forma determinística, Finalmente se extrae la información estadística de los resultados de las simulaciones. Los valores de índice de confiabilidad $\beta \mathrm{y}$ probabilidad de falla $p_{f}$ pueden ser calculados directamente de los resultados de los juegos de datos. Este método tiene como ventaja la simplicidad conceptual, pero requiere un gran número de juego de valores de la función de desempeño para obtener una precisión adecuada. A diferencia de otros métodos, la simulación de Monte Carlo no da luces sobre la contribución relativa de los parámetros aleatorios (Baecher y Christian, 2003).

Las simulaciones de Monte Carlo fueron implementadas en el programa matemático $M_{a t l a b}{ }^{\circledR}$, ya que se requiere un número significativo de iteraciones (en función del nivel de error y confiabilidad deseados) para obtener una respuesta cercana a la real. Para el caso del muro de contención se realizaron 100,000 simulaciones de Monte Carlo. La generación de números aleatorios del tipo normal y log-normal se hizo con las rutinas internas del programa.

Con los métodos de primer orden segundo momento (FOSM) y con el método de estimación puntual se obtiene tanto el valor esperado $E[g]$ como la varianza $V g]$ de la función de desempeño. Para estimar la probabilidad de falla $p_{f}$ es necesario suponer una función de densidad de probabilidad que tenga como parámetros el valor medio y la desviación estándar. Por lo que el factor de seguridad se calcula con el área bajo la curva entre - $\infty$ y 1 de la función de desempeño. El índice de confiabilidad $\beta$ se calcula como la relación entre la media y la desviación estándar de la función de desempeño.

Con el método de simulación de Monte Carlo se tiene directamente las realizaciones de la función de desempeño menor a 1. Si se dividen las anteriores entre el total de realizaciones, se calcula directamente la probabilidad de falla $p_{f .}$

\section{Caso de estudio}

Con el fin de presentar las metodologías de análisis basadas en la confiabilidad, se estudió la estabilidad de un muro de gravedad adyacente a un talud. El análisis consistió en determinar el ancho óptimo de la base del muro. Como criterio de diseño se consideró el factor de seguridad por capacidad portante, considerando el efecto del talud adyacente en la base, la acción de cargas excéntricas y la presencia del nivel freático.

El esquema del muro de contención que se analizará con los tres métodos de confiabilidad se presenta en la Figura 1, donde $\mathrm{H}=7 \mathrm{~m}, \mathrm{~B}^{\prime}=1 \mathrm{~m}, \mathrm{D}=0,5 \mathrm{~m}, \mathrm{~L}=2 \mathrm{~m}$. 


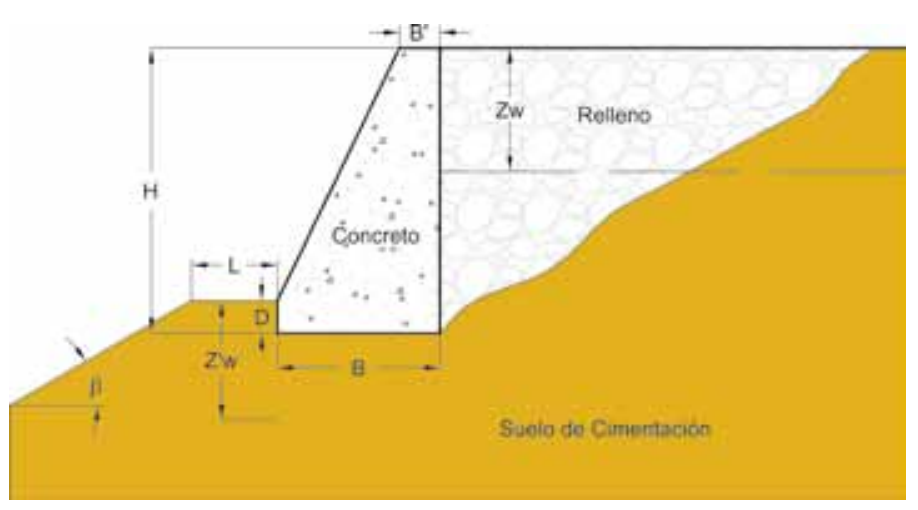

Figura 1: Geometría del muro de contención

Por facilidad de cálculo se supuso que los costos de construcción $C_{c}$ son proporcionales al volumen de concreto del muro $V_{c}, C_{c} \propto V_{c}$ y los costos que tendría la falla del muro en una vía de tráfico bajo son proporcionales a los costos de construcción, $C_{f} \propto 1.5 C_{c}$. Finalmente se calculó la probabilidad de falla $p_{f}$ del factor de seguridad FS para distintas bases del muro y se seleccionó en función de los costos de construcción y falla las dimensiones óptimas de la estructura de contención.

En la Tabla 1 se suministra como información básica la caracterización estadística de las propiedades mecánicas de los materiales de relleno y cimentación de la estructura de contención. Valores similares aparecen reportados en la literatura (Phoon, 2002; Baecher y Christian, 2003). En este punto es importante mencionar que no se han realizado estudios concienzudos de la caracterización probabilística de variables geomecánicas para materiales colombianos.

Tabla 1: Caracterización estadística de las variables geomecánicas

\begin{tabular}{cccc}
\hline & Media & $\mathrm{CV}(\%)$ & Distribución \\
\hline \multicolumn{4}{c}{ Suelo de cimentación } \\
$\varphi^{\prime}$ & 35 & 20 & Normal \\
$\mathrm{c}^{\prime}$ & $10 \mathrm{kPa}$ & 30 & Lognormal \\
$\gamma_{\text {st }}$ & $22 \mathrm{kN} / \mathrm{m}^{3}$ & 15 & Normal \\
$Z_{{ }_{\mathrm{w}}}^{\prime}$ & $3.0 \mathrm{~m}$ & 30 & Normal \\
$\beta$ & 20 & 10 & Normal \\
& & Relleno & \\
$\varphi_{r}^{\prime}$ & 30 & 20 & Normal \\
$c_{r}^{\prime}$ & 0 & & $\ldots .$. \\
$\gamma_{\mathrm{satr}}$ & $24 \mathrm{kN} / \mathrm{m}^{3}$ & 15 & Normal \\
$Z_{\mathrm{wr}}$ & $4.0 \mathrm{~m}$ & 30 & Normal \\
\hline
\end{tabular}

\section{Capacidad portante}

El dimensionamiento del muro de contención puede hacerse con la evaluación del factor de seguridad contra deslizamiento y contra volteo.
Sin embargo, el factor más crítico para este tipo de muros es la falla por capacidad portante, en especial cuando actúan fuerzas excéntricas y sísmicas sobre el muro. En el ejemplo planteado se diseñará el muro con el propósito que cumpla con las evaluaciones por capacidad portante.

La ecuación general de capacidad portante última para cimientos superficiales utilizada es la siguiente:

$$
q_{u}=c N_{c}^{*} F_{c} D_{c} i_{c}+q N_{q}^{*} F_{q} D_{q} i_{q}+\frac{1}{2} B \gamma N_{\gamma}^{*} F_{\gamma} D_{\gamma} i_{\gamma}
$$

donde $c$ es la cohesión, $q$ es el esfuerzo efectivo a nivel de desplante, $\gamma$ es el peso unitario del suelo por debajo del nivel de cimentación y $B$ es el ancho de la cimentación. $F_{c}, F_{q}, F_{\gamma}$ son los factores de corrección por forma del cimiento. Para el caso de estudio, los factores son iguales a 1.0 dado que la longitud del muro es lo suficientemente grande para considerar condiciones de deformacion plana (zapata corrida). $D_{c}, D_{q}, D_{\gamma}$ son factores de corrección por profundidad del cimiento. Estos factores no se tendrán en cuenta dado que se calcularán los factores de capacidad portante al cimiento corregidos por el efecto del talud adyacente al cimiento $N_{c}^{\prime}, N_{q}^{\prime}, N_{\gamma}^{\prime}$. En estos últimos ya se tiene en cuenta los efectos de la profundidad en las relaciones de área y longitud (Bowles, 1996). $i_{c}, i_{q}, i_{\gamma}$ son los factores de inclinación y excentricidad de la carga en el cimiento derivados por Vesic (1973) y publicados por Winterkorn y Fang (1975).

Los factores de capacidad portante $N_{q}$ y $N_{c}$ se determinaron con las fórmulas de Meyerhof (1963) y $N_{\gamma}$ con la fórmula de Vesic (1973):

$$
\begin{gathered}
N_{q}=e^{\pi \tan \delta} \tan ^{2}\left(45+\frac{\phi}{2}\right) \\
N_{c}=\left(N_{q}-1\right) \cot \phi \\
N_{\gamma}=2\left(N_{q}+1\right) \tan \phi
\end{gathered}
$$

Estos factores dependen exclusivamente del ángulo de fricción interna $\phi$ del suelo bajo la zapata.

\section{Reducción de los factores de capacidad portante por inclinación del terreno}

Una cimentación ubicada en proximidades de un talud debe ser analizada cuidadosamente. El hecho de tener una 
superficie inclinada en vez de una superficie horizontal por encima de la cimentación causa una reducción importante en su estabilidad. Cuando un cimiento se encuentra adyacente a un terreno inclinado los factores de capacidad portante se deben reducir a $N_{c}^{\prime}, N_{q^{\prime}}, N_{\gamma}^{\prime}$.

La corrección de los factores de capacidad portante se realizó con base en el procedimiento de Bowles (1996). Uno de los aportes del presente trabajo es la deducción geométrica de las ecuaciones de corrección de los factores de capacidad portante. Dichas deducciones parten del supuesto que la superficie de la cuña pasiva no alcanza a desarrollarse en su totalidad y que se inclina un ángulo de $45^{\circ}-\phi / 2$ con respecto a la superficie del talud.

Bowles (1996) recomienda que cuando se haga la corrección de los factores de capacidad portante por efecto de tener la cimentación en proximidades de un talud, los factores de profundidad $\left(D_{\mathcal{c}}, D_{q}, D_{\gamma}\right)$ se dejen iguales a 1.0, al igual que los factores de forma $\left(F_{c}, F_{q}\right)$. No es necesario incorporar los factores de profundidad ya que al hacer la relación entre las áreas de las cuñas pasivas con la condición del terreno horizontal y del terreno inclinado, se está considerando implícitamente el efecto de la sobrecarga por encima del nivel de desplante de la cimentación.

Para obtener $N_{c}$, se relacionan las longitudes de la superficies de falla que se forman cuando un cimiento está junto a un terreno inclinado $L_{1}=L_{2}+L_{e}$ (Figura 2) y cuando está sobre un terreno horizontal $L_{0}=r+L_{e}$ (Figura 3). Para derivar el factor $N_{q}$ es necesario relacionar las áreas de la sobrecarga en las dos situaciones anteriormente planteadas $A_{l}=a, b, c, d$ (Figura 2) y $A_{0}=a, b, c, d$ (Figura 3).

$$
N_{c}^{\prime}=N_{c} \frac{L_{1}}{L_{0}} \quad N_{q}^{\prime}=N_{q} \frac{L_{1}}{L_{0}}
$$

Al analizar cómo se forman las superficies de falla en un terreno horizontal e inclinado, se observa que la espiral se desarrolla en un ángulo $\left(90^{\circ}-\beta\right)$ y el ángulo $\left(90^{\circ}+\phi\right)$ de la cuña pasiva se mantiene constante (Figuras 4 y 5 ).

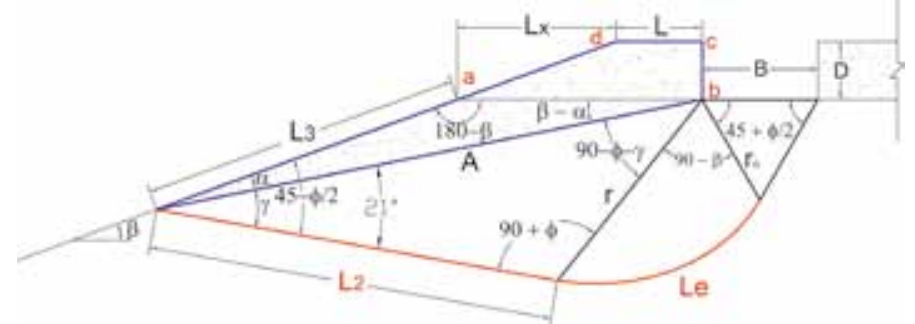

Figura 2: Geometría de la superficie de falla terreno inclinado

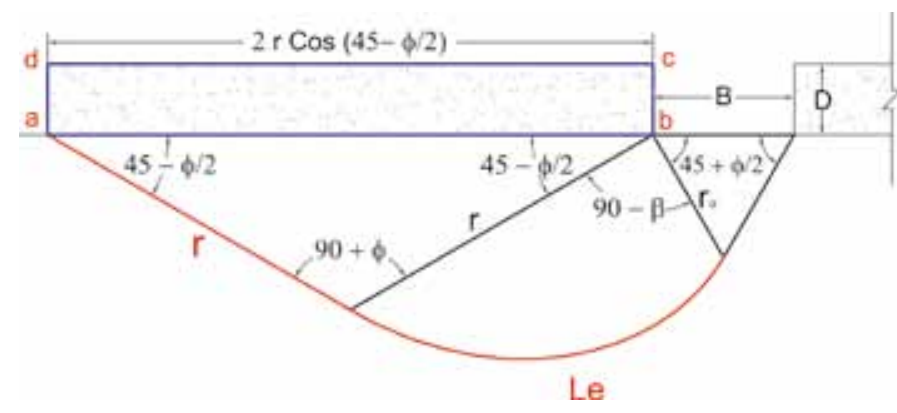

Figura 3: Geometría de la superficie de falla terreno horizontal

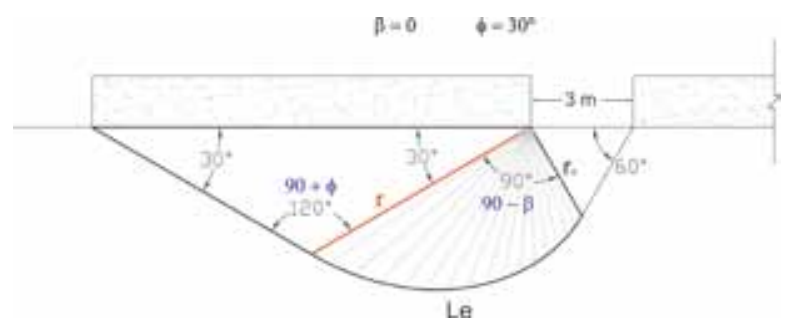

Figura 4: Evolución de la longitud de la espiral para una falla con terreno horizontal.

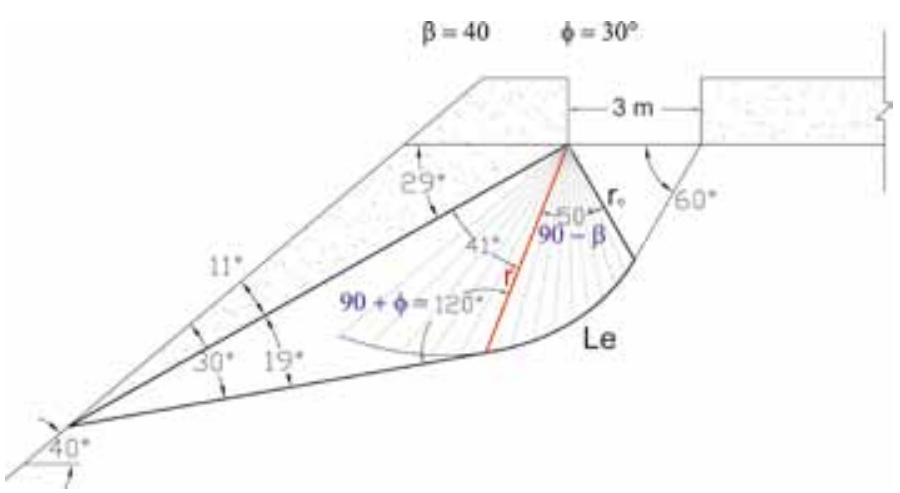

Figura 5: Evolución de la longitud de la espiral para una falla con terreno inclinado $\beta=40^{\circ}$

Partiendo de las hipótesis de los ángulos anteriormente planteadas se obtiene que el desarrollo y la longitud de la espiral vienen dados por:

$$
\begin{aligned}
r_{o} & =\frac{B}{2 \cos \left(45+\frac{\phi}{2}\right)} \quad r=r_{o} e^{\left(\frac{\pi}{2}-\beta\right) \tan \phi} \\
L_{e} & =\int_{0}^{\frac{\pi}{2}-\beta} \sqrt{r^{2}+\left(\frac{\mathrm{d} r}{\mathrm{~d} \theta}\right)^{2}} \cdot \mathrm{d} \theta \\
L_{e} & =\frac{e^{-\beta \tan \phi}\left|r_{o}\right|\left(e^{\frac{\pi}{2} \tan \phi}-e^{\beta \tan \phi}\right)}{\sin \phi}
\end{aligned}
$$

La longitud $\mathrm{L}_{2}$ de la cuña pasiva en el terreno inclinado se obtiene como:

$$
\begin{gathered}
\alpha+\gamma=45-\frac{\phi}{2} \\
\frac{\sin (180-\beta)}{A}=\frac{\sin a}{L+\frac{D}{\tan }} \\
\frac{\sin (90+\phi)}{A}=\frac{\sin \gamma}{r}
\end{gathered}
$$


Despejando $A$ de las ecuaciones(14)y(15) eigualando sellega a

$$
\frac{\sin (90+\delta) r}{\sin \gamma}=\frac{\sin (180-\beta)\left(L+\frac{n}{\tan 1}\right)}{\sin \alpha}
$$

$\alpha$ se despeja de (13) y se reemplaza en la ecuación (16):

$$
\frac{\sin \left(\left(45-\frac{\rho}{2}\right)-\gamma\right]}{\sin ?}=\frac{\sin (180-\beta)\left(L+\frac{D}{\tan 3}\right)}{\sin (90+\delta) r}
$$

Desarrollando $\sin [(45-\phi / 2)-\gamma]$ con la propiedad trigonométrica $\sin (a-b)=\sin a \cos b-\cos a \sin b$ y reemplazando en (17) se llega a:

$$
\cot \gamma=\frac{\frac{\sin (180-\beta)\left(L+\frac{D}{\tan \beta}\right)}{\sin \left(45-\frac{\delta}{2}\right)}+\cos \left(45-\frac{\rho}{2}\right)}{\sin \left(45-\frac{\delta}{2}\right)}
$$

Conociendo $\gamma$ de la ecuación (11) y por la ley de senos se determina $L_{2}$ :

$$
\begin{aligned}
& \frac{\sin \gamma}{r}=\frac{\sin (90-\phi-\gamma)}{L_{2}} \\
& L_{2}=\frac{\sin (90-\phi-\gamma) r}{\sin \gamma}
\end{aligned}
$$

La longitud $L_{l}$ para el terreno inclinado es:

$$
\begin{gathered}
L_{1}=L_{e}+L_{2} \\
L_{1}=e^{-3 \tan \phi} \frac{B}{2 \cos \left(45+\frac{\rho}{2}\right)} \frac{\left(e^{\frac{2}{2} \tan \phi}-e^{3 \tan \phi}\right)}{\sin \phi}+\frac{\sin (90-\phi-\gamma) r}{\sin \gamma}
\end{gathered}
$$

La longitud $L_{0}$ del terreno horizontal es:

$$
\begin{gathered}
L_{0}=L_{e}+r_{0} \\
L_{0}=\frac{B}{2 \cos \left(45+\frac{\phi}{2}\right)} \frac{e^{\frac{2}{2} \tan \phi}}{\sin \phi}+\frac{B}{2 \cos \left(45+\frac{\phi}{2}\right)} c^{\frac{B}{2} \tan \phi}
\end{gathered}
$$

El área $A_{l}$ de sobrecarga del terreno inclinado $a, b, c, d$ (Figura 2):

$$
\begin{aligned}
& A_{1}=\frac{\sin (90+\phi) r}{2 \sin \gamma}\left[L+\frac{D}{\tan \beta}\right] \sin \left(\beta-45+\frac{\phi}{2}+\gamma\right) \\
& +\frac{D}{2}\left[\left(L+\frac{D}{\tan \beta}\right)+L\right] D
\end{aligned}
$$

El área $A_{0}$ de sobrecarga del terreno horizontal $a, b, c, d$ (Figura 3):

$$
\begin{aligned}
& A_{\omega}=2 \cdot D \cdot r \cdot \cos \left(45-\frac{\delta}{2}\right) \\
& =\frac{D \cdot B}{\cos \left(45+\frac{g}{2}\right)} \cdot(j \tan \theta) \cos \left(45-\frac{\rho}{2}\right)
\end{aligned}
$$

Corrección de $N_{\gamma}$ para $K_{p}$ y $\beta<\phi-\beta$

$$
N_{\gamma}^{\prime}=\frac{N_{7}}{2}+\frac{N_{7}}{2}\left[\frac{K p_{\min }}{K p_{\max }}+\frac{L}{2 B}\left(1-\frac{K p_{\min }}{K p_{\max }}\right)\right]
$$

donde

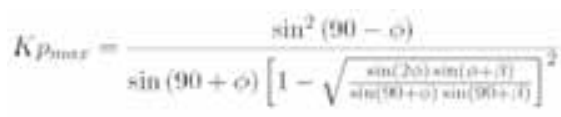

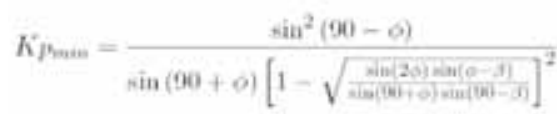

son los coeficientes de empuje pasivo máximo y mínimo de acuerdo a la teoría de Coulomb.

\section{Factores de seguridad}

Se define el factor de seguridad (FS) como:

$$
F S=\frac{q_{u}}{q_{\max }}
$$

donde $q_{u}$ es la capacidad portante última con todos los factores de corrección considerados en (7), $q_{\max }$ es la carga máxima aplicada a la cimentación y depende del grado de excentricidad de las fuerzas aplicadas sobre el muro. Teniendo en cuenta la excentricidad, se contemplan tres casos:

$$
\begin{aligned}
& \text { Si } e \leq B ; 6 \text { : } \\
& \psi_{\text {max }}=\frac{\sum F_{v}}{B}\left(1+\frac{6 \mathrm{c}}{B}\right)=\frac{\text { Peso del muro }}{B}\left(1+\frac{6 \mathrm{e}}{B}\right) \\
& \text { Si } B / 6<\mathrm{e}<B / 2 \text { : } \\
& q_{\max }=\frac{4 \sum F_{v}}{3 L(B-2 e)} \\
& \text { Si } e \geq B / 2 \text { : } \\
& q_{\max }=\frac{4 \sum F_{v}}{3 L(B-2 \cdot 0,499 \cdot B)}
\end{aligned}
$$

$F_{v}$ es la fuerza vertical equivalente al peso del muro:

$$
F_{t}=\gamma_{r}\left[B^{\prime} H+\frac{1}{2}\left(B-B^{\prime}\right)(H+D)\right]
$$

La excentricidad se define como $e=\mathrm{B} / 2-x$ donde $x$ es el punto de aplicación de la carga (momentos netos/fuerza vertical), y el subíndice hace referencia a las propiedades del material de relleno:

$$
\begin{aligned}
& \rightarrow H^{3}-12\left(B-B^{2}\right)\left(B+2 B^{\prime}\right)(D-H) \\
& 72\left(B^{\prime}(H-D)+B(D+H)\right)\left(\sin \varphi_{r}^{\prime}+1\right) \\
& +\frac{\left(7 H^{3}-20\left(H-Z_{w r}\right)^{3}+24 B^{2}(D-H)\right) \text { sin } \varepsilon^{\prime}}{72\left(B^{\prime}(H-D)+B(D+H)\right)\left(\text { sin } q_{r}^{\prime}+1\right)}
\end{aligned}
$$

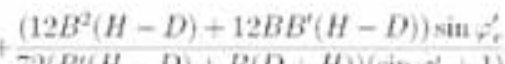

$$
\begin{aligned}
& 72\left(B^{\prime}(H-D)+B(D+H)\right)\left(\sin q_{r}^{\prime}+1\right)
\end{aligned}
$$

\section{Resultados}

Gracias a los programas matemáticos disponibles en el mercado (Maple, Mathematica, Derive), el desarrollo del método de primer orden segundo momento (FOSM) 
se reduce a plantear las ecuaciones paso por paso hasta llegar al ensamblaje de las derivadas de Taylor. En este ejemplo se utilizó el programa Mathematica.

El método de estimación puntual se implementó en una hoja de cálculo. Al tener 8 variables aleatorias (suelo de cimentación $\phi^{\prime}, \mathrm{c}^{\prime}, \gamma_{\mathrm{sat}}, Z^{\prime}{ }_{w}, \beta$; suelo de relleno: $\phi_{r}, \gamma_{\mathrm{r}}$, $Z_{w r}$ ) se deben generar $2^{8}=256$ combinaciones entre los parámetros para generar paquetes de variables de entrada a las ecuaciones de FS y MS. En la Tabla 2 se presenta un ejemplo con 4 combinaciones de parámetros de entrada. El signo + significa que se suma la desviación estándar a la media de la variable considerada y consecuentemente el signo - resta la desviación al valor medio.

Cada una de las 256 combinaciones de parámetros alimenta a la hoja de cálculo con el fin de obtener el FS para un ancho dado del muro. Al finalizar las 256 ejecuciones, se calcula el valor promedio y la desviación estándar del FS. Finalmente se calcula la $p_{f}$ como el área bajo la distribución normal con la media y desviación calculada de las 256 combinaciones.

Tabla 2: Ejemplo con 4 combinaciones de las variables aleatorias para el método estimación puntual

\begin{tabular}{ccccccccc}
\hline & $\varphi^{\prime}$ & $c^{\prime}$ & $\gamma_{\text {san }}$ & $Z_{w}^{\prime}$ & $\beta$ & $\varphi_{\mathrm{r}}^{\prime}$ & $\gamma_{\mathrm{r}}$ & $Z_{u r}$ \\
\hline 1 & + & + & + & + & + & + & + & + \\
& 42 & 13 & 25.3 & 3.9 & 22 & 36 & 27.6 & 5.2 \\
2 & - & - & - & - & - & - & - & - \\
& 28 & 7 & 18.7 & 2.1 & 18 & 24 & 20.4 & 2.8 \\
3 & + & + & + & + & - & - & - & - \\
& 42 & 13 & 25.3 & 3.9 & 18 & 24 & 20.4 & 2.8 \\
4 & - & - & - & - & + & + & + & + \\
& 28 & 7 & 18.7 & 2.1 & 22 & 36 & 27.6 & 5.2 \\
\hline
\end{tabular}

Para la simulación de Monte Carlo fue necesario acortar la generación de los números aleatorios distribuidos normal y log-normal a $3 \sigma$ para que los datos generados tuvieran sentido físico, es decir, con esto se está evitando que las propiedades del suelo generadas de forma aleatoria sean negativas. Se realizaron 100,000 simulaciones para anchos $B$ de muro variando entre $2.5 \mathrm{~m}$ a $10 \mathrm{~m}$. La rutina principal se implementó en Matlab.

En la Figura 6 se presentan los valores medios del FS y la $p_{f}$ del FS para la base del muro $B$ que varía de 2.5 a $10 \mathrm{~m}$ para los tres métodos considerados (estimación puntual, series de Taylor y simulaciones de Monte Carlo). Se observa que los FS para los tres métodos son muy parecidos. El FS varía entre 0 para $\mathrm{B}=2.5 \mathrm{~m}$, a 17 para $B=10 \mathrm{~m}$. Los valores de la $p_{f}$ difieren numéricamente, sin embargo la tendencia es similar. Para los tres métodos se observa un comportamiento asintótico para bases de muro mayores a $6 \mathrm{~m}$. Es decir que no cambia significativamente la $p_{f}$ aunque se aumente el ancho de la base.
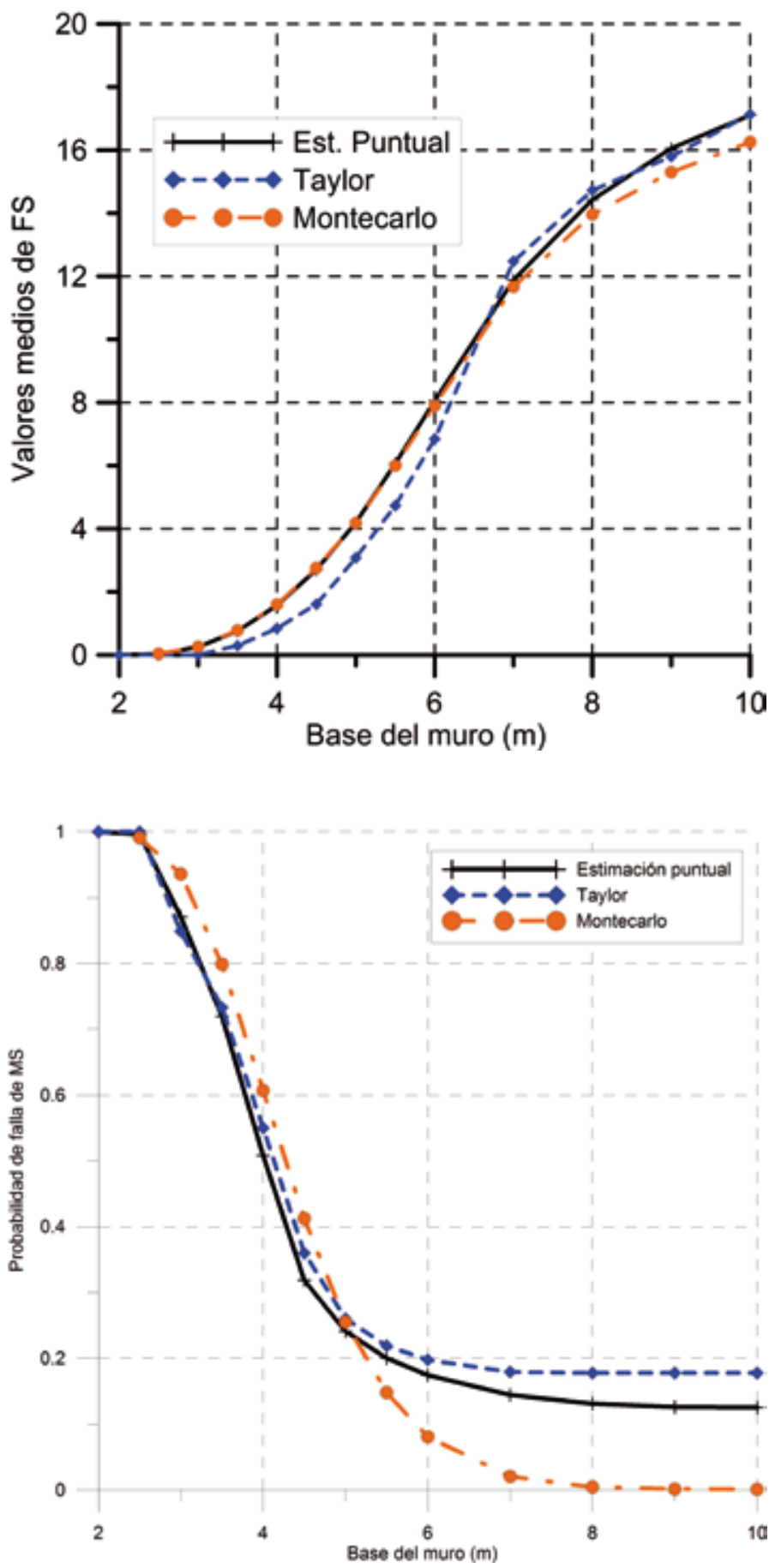

Figura 6: FS y $p_{f}$ del FS en función de la base B del muro

Las probabilidades de falla del FS no se distribuyen con una función normal. Esto se pudo observar al graficar los histogramas de frecuencia de los factores de seguridad en las simulaciones de Monte Carlo. Los factores de seguridad para estos casos se pueden ajustar mejor a una distribución de tipo Log-Normal, como se muestra en la Figura 7. 

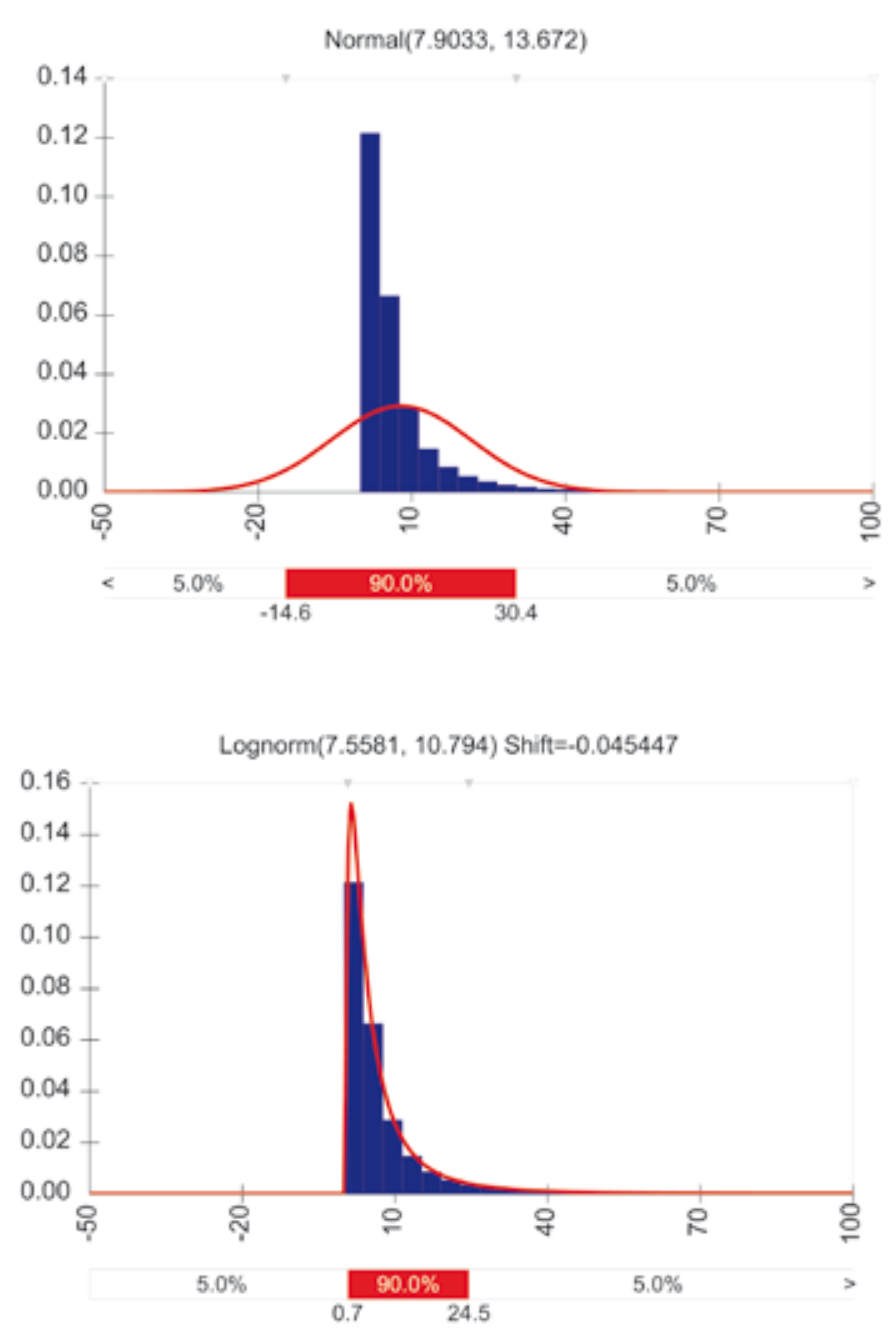

Figura 7: Ajuste del FS con el método de Monte Carlo para $B=6.0 \mathrm{~m}$ con la función de densidad de probabilidad normal y log-normal.

Para obtener el ancho de muro $B$ óptimo se calculó la suma del costo de falla y el costo de construcción por los tres métodos de confiabilidad mencionados. En la Figura 8 se observa la función de utilidad a minimizar expresada en términos de la suma de los costos de falla más los costos de construcción.

Para obtener el ancho del muro $B$ óptimo minimizando la función de utilidad, se analizaron los resultados con los tres métodos y se escogió el $B$ óptimo por el método de Monte Carlo, ya que éste permite conocer el comportamiento de la probabilidad de falla tanto en los valores medios como en los valores extremos del ancho del muro. Además este método permite obtener la función empírica de distribución de la $p_{f}$ El ancho $B$ de la base del muro encontrado con el valor mínimo de la función de utilidad para una vía de bajo tráfico es de $B=6.0 \mathrm{~m}$. a)

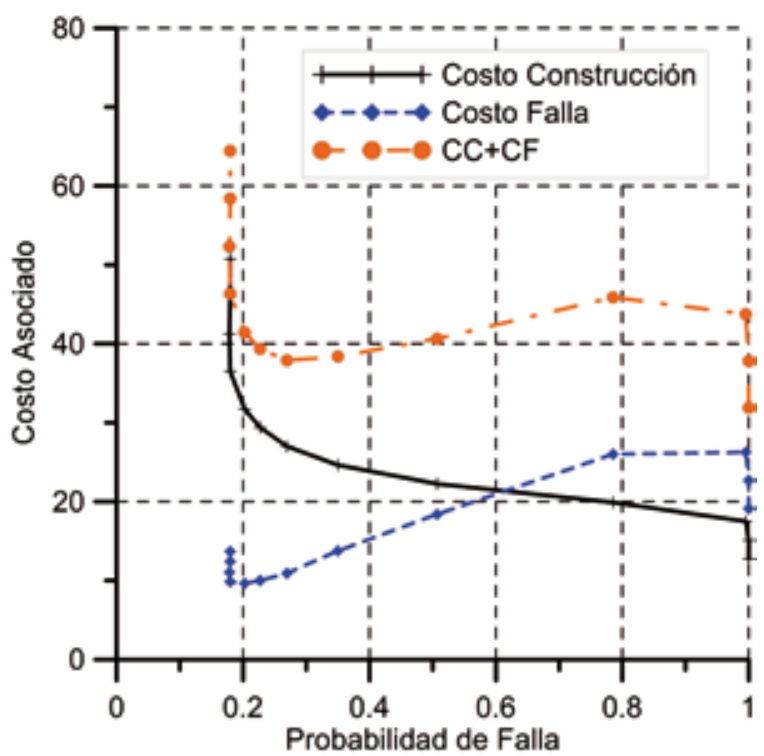

b)

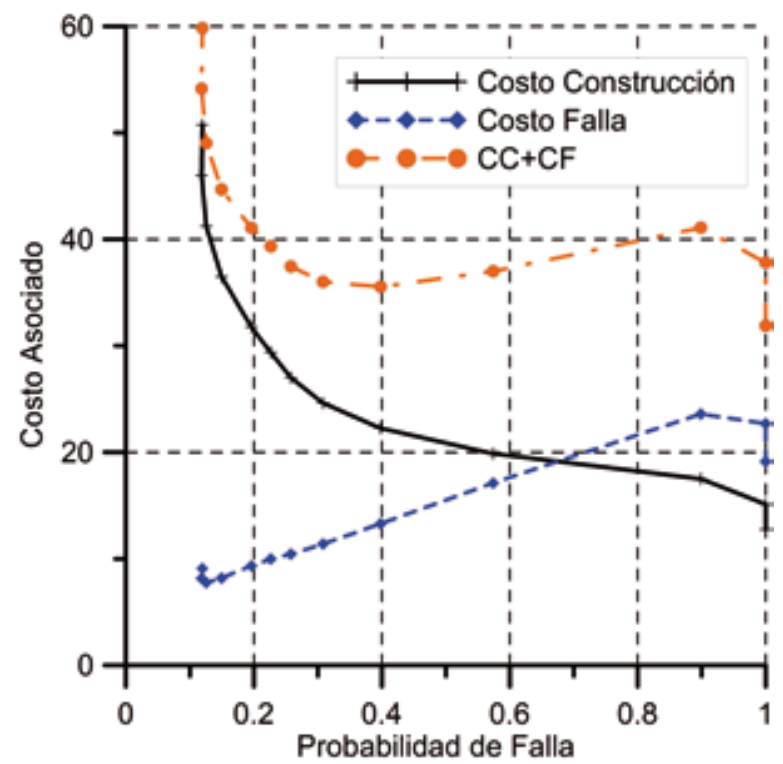

c)

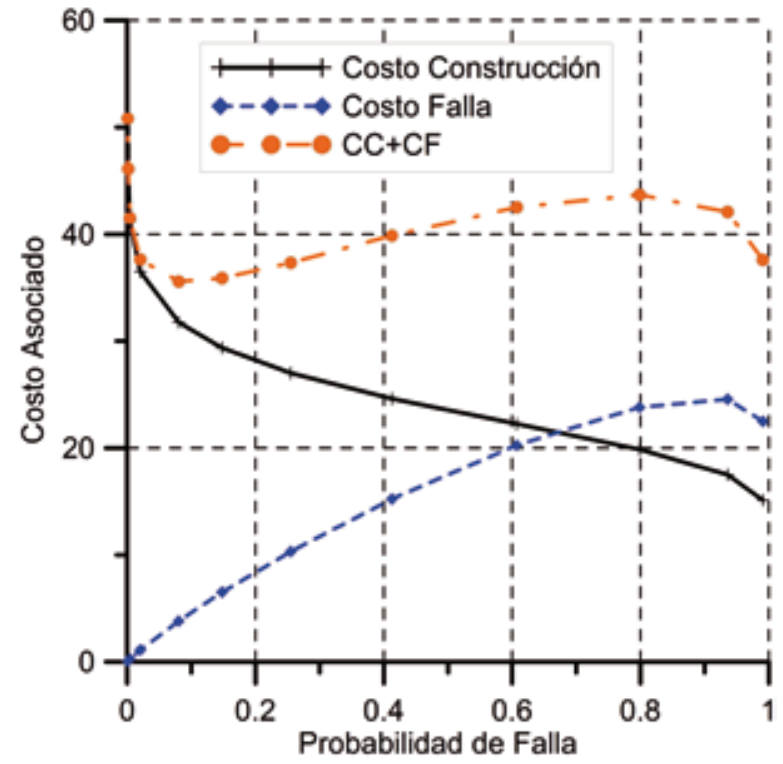

Figura 8: Costo de falla más costos de construcción para la vía de bajo tráfico, a) método de Taylor; medio, b) método de estimación puntual y c) método de Monte Carlo. 
A partir de los resultados de las simulaciones de Monte Carlo se analizó la relación entre el FS y la $p_{f}$ del FS para cada una de las bases analizadas (Figura 9). Se observa claramente que resulta erróneo suponer que un $\mathrm{FS}=1$ es equivalente a una $p_{f}$ del $100 \%$. Para el ejemplo analizado, un $\mathrm{FS}=3.0$ que es el que recomienda la literatura para el chequeo por capacidad portante de un muro, equivaldría a una $p_{f} \approx 40 \%$. La probabilidad de falla es la que se debería considerar en el momento de tomar una decisión para diseño.

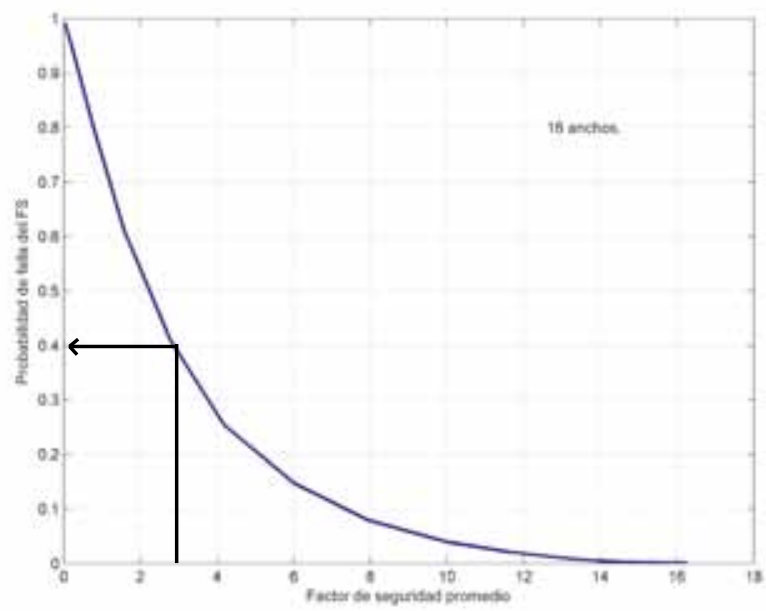

Figura 9: Probabilidad de falla del FS versus FS promedio con el método de Monte Carlo

\section{Discusión}

La probabilidad de falla no disminuye indefinidamente con el aumento de la base del muro si se tiene en cuenta los métodos de Taylor y de estimación puntual. Por el contrario, la simulación de Monte Carlo sí muestra que la probabilidad de falla tiende a cero con el aumento de la base del muro. Este comportamiento se puede explicar mencionando que el método de simulación de Monte Carlo permite obtener resultados de probabilidad de falla para valores extremos del ancho de la base del muro. Los métodos de las series de Taylor y estimación puntual utilizan parámetros de entrada que tienden a distribuirse en proximidades a los valores medios. Esto hace que el comportamiento sea representativo en este mismo rango.

A medida que el ancho de la base del muro aumenta, la diferencia en la probabilidad de falla calculada por los tres métodos aumenta, siendo asintótico para valores mayores a 8 metros (Figura 6). El método de estimación puntual parte del supuesto que la función de estado límite (FS) se distribuye normalmente. Resultados obtenidos para el caso del análisis del muro de contención indican que el FS se ajusta mejor a otro tipo de distribución de probabilidad, similares a una función exponencial o Log-Normal.

Las soluciones con series de Taylor entregan resultados que se podrían considerar más ajustados a los valores centrales, ya que para obtener la desviación estándar se evalúan las derivadas en los valores medios. Sin embargo, el método se vuelve dispendioso cuando las variables aleatorias hacen parte de formulaciones complejas como en el caso de los factores de capacidad portante.

El método de estimación puntual podría no siempre dar un resultado ajustado a las observaciones del comportamiento de las variables aleatorias ya que a pesar que dichas variables puedan ajustarse a una distribución normal, al ser "filtradas" por medio de una ecuación (e.g. capacidad portante, asentamientos) la distribución de la función de desempeño no va a seguir teniendo una distribución normal.

El método de Monte Carlo puede dar una buena idea de la incertidumbre de un sistema con variables aleatorias. Su gran desventaja es la necesidad de realizar un alto número de ejecuciones para obtener un muestreo significativo del espacio probable de ocurrencia de las variables. Mayor número de simulaciones produce mejores resultados pero a un costo computacional bastante elevado.

El método de Monte Carlo permite obtener valores representativos de probabilidad de falla aún para valores extremos. En este caso los valores extremos se refieren a anchos de muro muy pequeños o muy grandes. La probabilidad de falla para casos extremos calculada con el método de estimación puntual y por el método de las series de Taylor parece "saturarse" con el método de simulación de Monte Carlo, a medida que el ancho de una cimentación aumenta, la probabilidad de falla es asintótica hacia cero.

De otro lado, y con los otros dos métodos, la probabilidad de falla es asintótica a valores mayores a cero (lo cual no resulta ser lógico). La diferencia en los resultados en valores extremos puede deberse a que la función de distribución usada para modelar la probabilidad de falla no se ajusta a los datos de factores de seguridad. Como la probabilidad de falla calculada de las simulaciones de Monte Carlo se obtiene por medio de un análisis de 
frecuencias, la probabilidad de falla no depende de una suposición adicional sino que la simulación de Monte Carlo entrega directamente la función de distribución empírica.

\section{Conclusiones}

Los factores de seguridad que rigen en la práctica diaria del diseño en la ingeniería son por lo general determinados por la experiencia y criterio del ingeniero geotecnista. Un factor de seguridad único no permite distinguir entre las incertidumbres del modelo y la variabilidad del material estudiado y de las cargas actuantes. Las metodologías basadas en el análisis de confiabilidad permiten aislar cada componente y estudiar de manera objetiva la influencia de su variabilidad en la respuesta general del sistema.

Es bien conocida la deficiencia de estudios sistemáticos de las funciones de densidad de probabilidad para variables geotécnicas. Tomar los valores reportados en la literatura puede ser la única opción cuando se van a realizar análisis de confiabilidad. Sin embargo es necesario tener cuidado con aquellas propiedades geomecánicas cuyo ambiente geológico y de formación de los suelos en el trópico no se hayan investigado en otras latitudes, como por ejemplo la permeabilidad o parámetros de resistencia en suelos residuales.

La cantidad de variables aleatorias incluidas en la función de desempeño afectan directamente el costo computacional o el tiempo invertido en las simulaciones (caso de Monte Carlo), resultando muchas veces este método impráctico.

Se recomienda determinar de antemano cuáles variables geotécnicas presentan mayor variabilidad con respecto a otras para de esa forma poder restringir el tamaño del problema y en consecuencia disminuir el tiempo de simulación. Por ejemplo, la variabilidad de la base de una cimentación superficial es menor a la variabilidad del diámetro de un pilote preexcavado y fundido in situ. Estudios de probabilidad de falla asociados con costos de construcción y costos de falla permiten tener un importante insumo para poder determinar las dimensiones óptimas de estructuras geotécnicas. Es decir, análisis como los mostrados en este artículo permiten tomar decisiones informadas. Situación que no se presenta cuando se realizan análisis convencionales deterministas de factor de seguridad.

\section{Agradecimientos}

El primer y segundo autor expresan sus agradecimientos a Colciencias-Icetex, alCentrodeexcelenciaenComplejidad CeiBA, al Centro de Investigaciones de la Facultad de Ingeniería (CIFI) de la Universidad de Los Andes y a la PontificiaUniversidad Javeriana - Colombia-porel soporte económico para el desarrollo del presente trabajo.

\section{Referencias}

Baecher, G. and Christian J. (2003). Reliability and statistics in geotechnical engineering. John Wiley. Chichester, England. $1^{\circ}$ edición

Berdugo, I. R. (2000). Manual de diseño geotécnico de cimentaciones. Pontificia Universidad Javeriana, Bogotá, $1^{\circ}$ edición.

Bowles, J. (1996). Foundation analysis and design. McGrawHill, Singapore, $5^{\circ}$ edición.

Meyerhof, G. (1963). Some recent research on the bearing capacity of foundations. Canadian Geotechnical Journal, 1(1):16-26.

Phoon, K. (2002). Potential application of reliability-based design to geotechnical engineering. In 4 th Colombian Geotechnical Seminar, 1-24.

Rosenblueth, E. (1975). Points estimates for probability moments. National Academy of Science 72 , 3812-3814.

Sanchez-Silva, M. (2005). Introducción a la confiabilidad y evaluación de riesgos. Teoría y aplicaciones en ingeniería. Universidad de Los Andes, Facultad de Ingeniería, $1^{\circ}$ edición.

Vesic, A. (1973). Analysis of ultimate loads on shallow foundations. Journal of the Soil Mechanics and Foundations Division ASCE, 99(SM1):45-71.

Winterkorn, H.F. and Fang, H. Y. (1975). Foundation Engineering Handbook. Van Nostrand Reinhold Company Inc, New York, $1^{\circ}$ edición. 